\title{
Implementation of Boundary Alert system for Fishermen
}

\section{S. Niveda ${ }^{a}$, S. Srinitha ， A.Sivasakthi and S. Rangeetha ${ }^{d}$}

aAssistant Professor, Electronics and Communication Engineering, Sri Ramakrishna Engineering College, Coimbatore, India.

${ }^{\mathbf{b}}$ Assistant Professor, Electronics and Communication Engineering, Sri Ramakrishna Engineering College, Coimbatore, India.

'Assistant Professor, Biomedical Engineering, Sri Ramakrishna Engineering College, Coimbatore, India.

dAssistant Professor, Electronics and Communication Engineering, Sri Ramakrishna Engineering College,

Coimbatore, India.

Article History: Received: 10 January 2021; Revised: 12 February 2021; Accepted: 27 March 2021; Published online: 20 April 2021

\begin{abstract}
Fishing is one of the main occupations of the people who live near the coastal areas. In order to satisfy their daily requirements, fishermen need to get more fish. Once fishermen are not satisfied at a certain place, they move to further places to acquire more fish. Sometimes, without their knowledge they cross the border. Due to this, the navy of the other country arrests him and sentence them to prison. This happens because there is no awareness of boundary for the fishermen. In order to create an awareness for the fishermen, we proposed a system. Our proposed systemwill be an alternate source for avoiding such kind of accidents andalert the fishermen about the border area. In addition to that, in our project we implemented automatic turning of the boat in desired direction even when the alarm system fails to alert the fishermen by using RFtechnology. This is done byfixing the receiver in the boat and the transmitter has to be placed before 100 meters from the IMBL (International Marine time Boundary Line). This can be used to avoid crossing the boundary.
\end{abstract}

Keywords: RF Transmitter, RF Receiver, Alarm Buzzer, DC Motor, ArduinoATmega328

\section{Introduction}

The Marine time border is not familiar as land borders. We all know that for people in coastal areas fishing is one of the main occupations. The navy of the other country arrests Indian fishermen when they cross the boundary line of their border.Usually charged of trespassing the maritime boundary and sentenced them to prison (Kumar \& Kumar, 2010). This is happening because there is no awareness of boundary for the fishermen. In order to create an awarenessfor the fisherman, an alarm system is proposed in the boat. Though there are many proposed systems they are not viable due to its high cost. The existing proposed system uses GPS(Global positioning system) (Isaac \& Kingsley, 2015); (Karuppasamy et al., 2017) and GSM (Global system for Mobile Communication) technologies, which is high cost and also cannot be afforded by poor fishermen. Extreme atmospheric conditions, such as geomagnetic storms, can also cause problem. In addition to that, GPS is associated with the mapping technology which may not be up to date and cause errors in navigational.There are fewerrors like Orbital errors which is also called as ephemeris errors, these are reported for inaccuracy of the satellite's location.Many border alert systems have been put forward based on GPS technology. This Project comes with the technology of Radio Frequency (RF) and is cost efficient and can be afforded easily by fishermen (Karthikeyan et al., 2012). For the safety of the fishermen here we used an alarm system to notify before the border and in addition to it if alarm system fails the boat itself changes to the desired direction. Thus, the life of the fishermen is secured (Bolong et al., 2013).

The objective of this project is to design an automatic border alert system using RF technology (Karthik et al., 2017); (Nagaraj Balakrishnan et al., 2017). Here we place Receiver in boat and the Transmitter in Buoy which is placed 100 meters before the border. Hence when the receiver comes into contact with the transmitter the voice play back module (alarm buzzer) in the boat alarms and the boat gets turned to 180 degree (Kumar et al., 2016); (Krishnamoorthy et al., 2017). So, that we can avoid trespassing the International Marine Boundary Line (IMBL).

\section{Methods}

\section{A. Principle of Operation}

The step by step connection procedure is given as follows; the power supply for this project is given using a $9 \mathrm{v}$ or $12 \mathrm{v}$ battery. For this project, battery of $12 \mathrm{v}$ is used.The supply is given to the L293D motor driver and as the Arduino requires a 5v power supply, it is connected to the $5 \mathrm{v}$ pin in the dc motor driver L293D.The input pins 9 and 10 of the Arduino are connected to the input pins 1 and 2 to control the left motor driver L293D.The input pins 11 and 12 of theArduino are connected to the input pins 3 and 4 to control the right motor L293D.The 8th pin of the Arduino is connected to the RF receiver module (HT12D). The 13th pin of the Arduino is connected to the voice playback (ISD1820). The above setup is placed in a boat. The Transmitter module is placed on BUOY (figure 2). This RF transmitters is placed 100 meters before the border. The voice playback (ISD1820) is used by pre-recording an alarm tone or voice, which is done by holding the rec button. The recording of the voice playback is (ISD1820).

B. Working of the Circuit

The step by step working procedure is given below:

- The active-HIGH REC is an input record signal.

- HIGH in REC represents the module starts recording.

- The recording duration is calculated only when the pin is in HIGH. 
- The playback (PLAYL or PLAYE) signalis controlled and having precedence on both by REC.

- The input of microphone transfers to the onchip preamplifier. This is called as MIC input.

- To enable the speaker, the mini jumper is connected to themicrophone. To play the recordings endlessly connect to mini jumper.

- Operational frequency of this model is at $433 \mathrm{MHz}$. In transmission and receiving part RF signal is travelled even when there is disturbance also.

- The serial data is received to RF transmitter and transmitted to the antenna receiver connected to the 4 th pin.

- Transmitter has no power supply when logic 0 is applied. It is considered as OFF state.

- Transmitter has high-power supply in the range of $4.5 \mathrm{~mA}$ with $3 \mathrm{~V}$ voltage supply when logic 1 is applied. It is considered as $\mathrm{ON}$ state.

- The Arduino is used to control the motors by code when it approaches the border.

- When the boat's RF receiver nears the transmitter, the input becomes high which makes the voice playback to be activated and will control the boat's motor by turning to $180^{\circ}$ and gets stopped.

- When input is low i.e., when the boat is not near the border, the input in the receiver is low, so the voice playback is not activated.

\section{Components}

The implementation device contains simple components like (Sivaramaganesh et al., 2014):

- Arduino.

- RF Transmitter (HT12E).

- RF Receiver (HT12D).

- L293D Motor Driver.

- Voice Playback
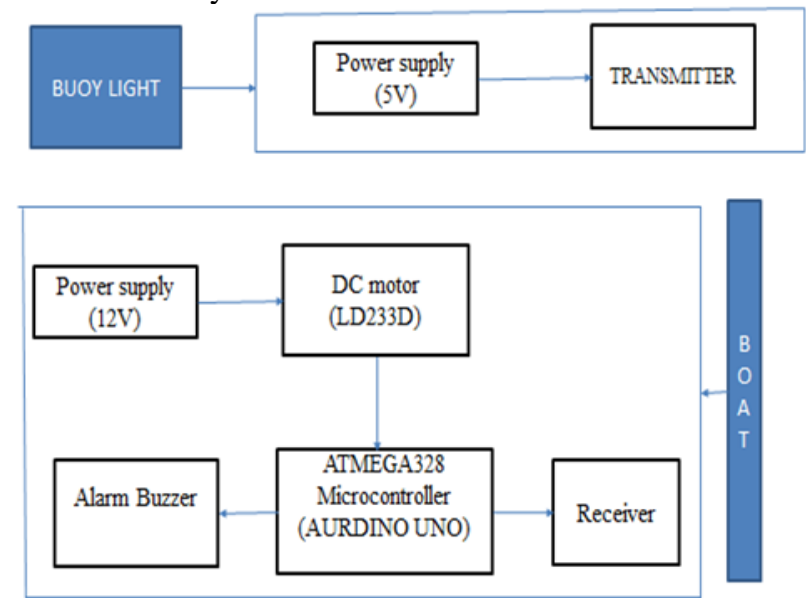

Figure 1. Block Diagram

The components are connected as per the block diagram figure 1 and the power supply is given. The device starts to work with their specific functions and indicate border alerting. It is a very simple devicewith reduced cost and setup time.

RF Transmitter

Basically, the RF modules are of frequency 433MHz RF transmitter (encoder) and receiver (decoder) modules. Here HT12E RF transmitter is an encoder integrated circuit of 2, 12 series of encoders. Applications like remote control uses series of decoders paired with 2, 12. Circuits like infrared and RF are mainly used in interfacing the circuits. Parallel input are converted into serial output by HT12E. For transmission 12-bit parallel data encodes into serial through an RF transmitter. For encoding there are 12 bits. Which are divided into 4 data bits and 8 address bits.An enable pin for transmission in HT12E is in active low. Transmission enable (TE) pin is used for receiving triggered signal and the header bits are transmitted along with programmed addresses/data in RF or infrared medium. Transmission enable upon a transmission cycle of 4 word in HT12E. When the TE is low the cycle will be repeated continuously. As soon as TE returns to high, the encoder output completes its final cycle and then stops (https://embetronicx.com/tutorials/microcontrollers/lpc2148/rf-module-interfacing-withlpc2148/); (https://embetronicx.com/tutorials/microcontrollers/lpc2148/rf-module-interfacing-withlpc2148/).Figure 2 shows the RF transmitter. 


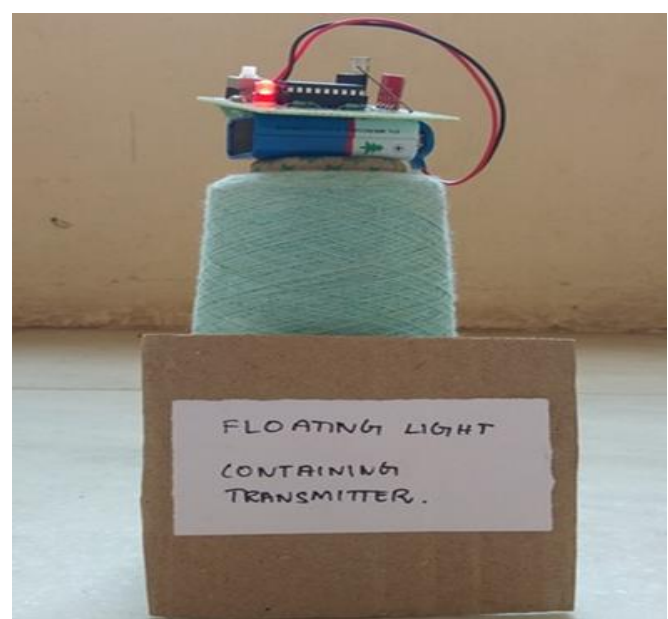

Figure 2. RF Transmitter

\section{RF Receiver}

Here HT12D RF receiver is a decoder integrated circuit of 2, 12 series of decoders. Applications like burglar alarm, car door controller, security system and many are major used for control system is series of decoders. The circuits like RF and infrared are interfaced with the help of it and its main purpose too.

Encoders are of 2, 12 pair of series. The same number of addresses and data format is for the pair of chosen encoder/decoder. In other words, serial input converts into parallel outputs by HT12D. The RF receiver decodes the serial addresses and data received in it,output data pins receives from parallel data. For continuously 3 times the serial data input is compared to local addresses. When no error or unmatched codes are found the input data code decoded. When a high signal is indicated at VT pin it is called Valid Transmission (VT) pin. In HT12D is able of decode bits with 12 bits. Which are 4 are data bits 8 address bits. Output remain unchanged in 4-bit latch type until when new data received. Figure 3 shows the RF receiver.

The prototype model shows the below connection of the device.

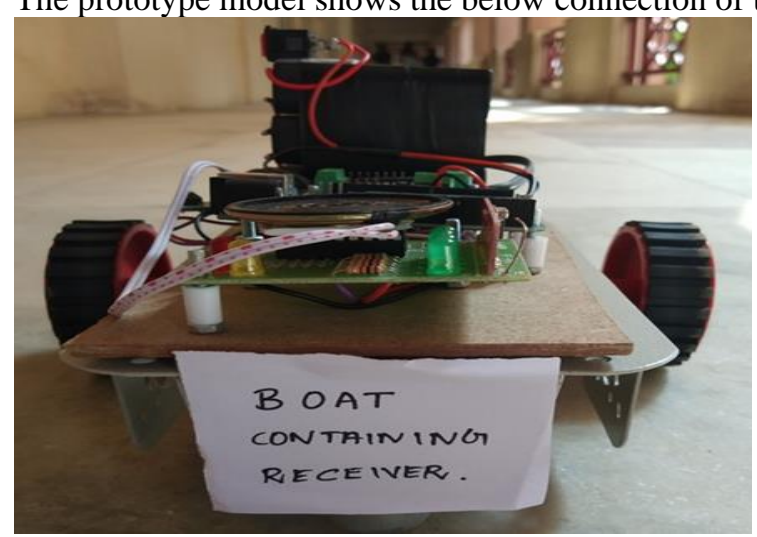

Figure 3. RF Receiver

\section{Implementation}

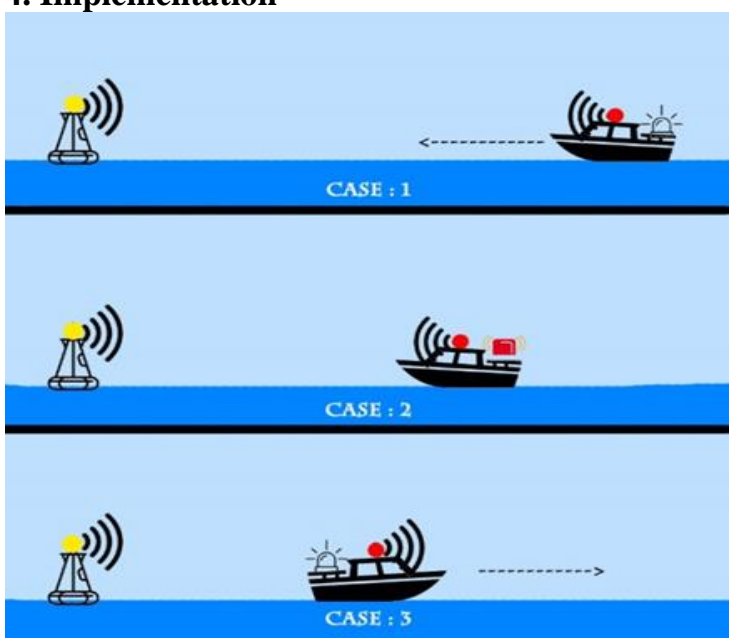

Figure 4. Implementation Model 
Figure 4 shows the implementation model. Let us consider this scenario. Here in the above image the RF Transmitter is placed in the Buoy which is placed before the border. Receiver is placed in the boat which is implemented with voiceplayback.

Case1:

In this case the boat is far away from the Buoy. So, the boat moves forward.

Case 2:

In this case the boat which consists of RF Receiver comes in contact with the RF Transmitter placed in the Buoy. Simultaneously the alarm starts to alert the fishermen.

Case 3:

When the fishermen reach a distance before the border, they are alerted by alarm system and in addition to that the boat itself changes its direction. Thus, the life of the fishermen is secured.

\section{Future Scope}

For the prototype model we have used RF technology. For real time application we can go with Lo-Ra (Long Range) technology.In IoT connections and Industry 4.0 are considered to be the fastest growing category of deployments in the field of wireless IoT connectivity. It's here that we meet Lo-Ra, WAN more specifically in the category of wireless wide area network technologies that need to cover a long range but need little power and low bandwidth, commonly known as LPWA networks or LPWAN (low-power, wide-area networks).

\section{Conclusion}

The growth of technologies has given rise to new possibilities in terms of securitysystem. The system that we proposed provides awareness to the fishermen about the International Marine Boundary Line (IMBL) with the help of RF Transmitter and Receiver. The efforts made in this paper are indented to improve the awareness by intimating the border before 100 meters, and thereby we can maintain a good relationship between the neighbor countries.

\section{References}

1. Kumar, K. S., \& Kumar, M. K. S. (2010). Design of low cost maritime boundary identification device using GPS system. International Journal of Engineering Science and Technology, 2(9), 4665-4672.

2. Karthikeyan, R., Dhandapani, A., \& Mahalingham, U. (2012). Protecting of fishermen on Indian maritime boundaries. Journal of Computer Applications, 5(3), 231-235.

3. Bolong, J., Osman, N., Omar, S. Z., D'Silva, J. L., \& Mohamed, H. A. (2013). Development of Instrument on Youth Fishermen's Readiness to Use Geographical Positioning Systems in Their Fishing Operations. Life Science Journal, 10(3), 2153-2157.

4. Sivaramaganesh, M., Ramya, M., Gowtham, V., Bharathi, T., \& Jeevitha, G. (2014). Implementation of maritime border alert system. International journal of innovative research in electrical, electronics, instrumentation and control engineering, 2(3), 1254-1257.

5. Isaac, J., \& Kingsley, H. E. (2015). Advanced border alert system using GPS and with intelligent Engine control unit. Int. J. Electr. Comput. Eng.(IJECE), 1(4), 11-14.

6. Karuppasamy, K., Jagadheesh, K., Nehru, K., Sindhu Meena. K., \& Vignesh, S. (2017). Providing a border alert system for fishermen by using gps\&gsm technology in wireless sensor networks. International Research Journal of Engineering and Technology (IRJET), 04 (3), 1904- 1907.

7. Karthik, R., Ranjith, S., Shreyas, S., \& Kumar, P. (2017). Automatic Border Alert System for Fishermen using GPS and GSM techniques. Indonesian Journal of Electrical Engineering and Computer Science, 7(1), 84-89.

8. Nagaraj Balakrishnan, R. S., Arunkumar, R., \& MS, P. (2017). A survey on border alert systems for fishermen. Pak. J. Biotechnol. Vol, 14(4), 829-831.

9. https://embetronicx.com/tutorials/microcontrollers/lpc2148/rf-module-interfacing-with$1 \mathrm{lpc} 2148 /$

10. https://embetronicx.com/tutorials/microcontrollers/lpc2148/rf-module-interfacing-withlpc2148/

11. Kumar, R. D., Aldo, M. S., \& Joseph, J. C. F. (2016). Alert system for fishermen crossing border using Android. International Conference on Electrical, Electronics, and Optimization Techniques (ICEEOT), pp. 4791-4795. 
12. Krishnamoorthy, E., Manikandan, S., MohamedShalik, M., \& Samy, S. M. (2017). Border alert system and emergency contact for Fisherman using RSSI. International Conference on Information Communication and Embedded Systems (ICICES), pp. 1-5. 